\title{
Single-shot soft $x$-ray laser linewidth measurement using a grating interferometer
}

\author{
Y. Wang, ${ }^{1,2}$ L. Yin, ${ }^{1,2}$ S. Wang, ${ }^{1,2}$ M. C. Marconi, ${ }^{1,2}$ J. Dunn, ${ }^{4}$ E. Gullikson, ${ }^{5}$ and J. J. Rocca ${ }^{1,2,3, *}$ \\ ${ }^{1}$ NSF ERC for Extreme Ultraviolet Science and Technology, Colorado State University, Fort Collins, \\ Colorado 80523, USA \\ ${ }^{2}$ Electrical and Computer Engineering Department, Colorado State University, Fort Collins, \\ Colorado 80523, USA \\ ${ }^{3}$ Physics Department, Colorado State University, Fort Collins, Colorado 80523, USA \\ ${ }^{4}$ Lawrence Livermore National Laboratory, Livermore, California 94550, USA \\ ${ }^{5}$ Lawrence Berkeley National Laboratory, Berkeley, California 94720, USA \\ ${ }^{*}$ Corresponding author: rocca@engr.colostate.edu
}

Received September 13, 2013; accepted October 11, 2013;

posted October 23, 2013 (Doc. ID 197584); published November 21, 2013

\begin{abstract}
The linewidth of a $14.7 \mathrm{~nm}$ wavelength Ni-like Pd soft x-ray laser was measured in a single shot using a soft x-ray diffraction grating interferometer. The instrument uses the time delay introduced by the gratings across the beam to measure the temporal coherence. The spectral linewidth of the $4 \mathrm{~d}^{1} \mathrm{~S}_{0}-4 \mathrm{p}^{1} \mathrm{P}_{1}$ Ni-like Pd lasing line was measured to be $\Delta \lambda / \lambda=3 \times 10^{-5}$ from the Fourier transform of the fringe visibility. This single shot linewidth measurement technique provides a rapid and accurate way to determine the temporal coherence of soft x-ray lasers that can contribute to the development of femtosecond plasma-based soft x-ray lasers. (C) 2013 Optical Society of America

OCIS codes: (140.7240) UV, EUV, and X-ray lasers; (340.7480) X-rays, soft x-rays, extreme ultraviolet (EUV); (120.3180) Interferometry.

http://dx.doi.org/10.1364/OL.38.005004
\end{abstract}

There is great interest in the generation of intense, coherent soft x-ray beams for scientific and technical applications. The temporal coherence length is an important parameter in applications, such as holography [1,2],

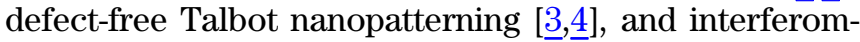
etry [ $\underline{5}-11]$. Inversely proportional to the linewidth, the corresponding transform limit determines the minimum pulse duration that can be achieved. Progress toward subpicosecond soft x-ray laser (SXRL) pulses $[12,13]$ requires accurate measurements of the spectral characteristics of these laser sources. However, these measurements are challenging because the linewidths of atomic soft x-ray lasers are very narrow, typically $\Delta \lambda / \lambda<$ $5 \times 10^{-5}$ for transient collisional lasers $[\underline{14}, \underline{15}]$, requiring a resolution that greatly exceeds that of commonly available spectrometers. In collisional soft x-ray lasers, the atomic linewidth is dominated by a combination of Doppler and collisional broadening and is affected by gain-narrowing effects during the amplification and by potential re-broadening during gain-saturation [16]. SXRL linewidth measurements have been performed using a high-resolution spectrometer [16] or using the difference in path length between the two arms of an interferometer $[14,15,17]$. Koch et al. built an $8 \mathrm{~m}$ long spectrometer with a resolution as high as $35,000\left(\Delta \lambda / \lambda=2.9 \times 10^{-5}\right)$ to measure the linewidth in collisional quasi-steady state SXRLs [16]. Measurements for transient collisional lasers were performed using an amplitude division Michelson interferometer that uses a thin multilayer membrane as beam splitter [14] and using a wavefront division interferometer consisting of a pair of slightly tilted dihedrons [15]. The measurement of SXRL linewidths with these interferometers requires numerous laser shots to obtain the visibility curve, which makes the measurement dependent on the stability of the source. Herein we report the first single-shot measurement of the linewidth of a transient SXRL and the demonstration of a soft xray Mach-Zehnder interferometer that utilizes the time delay introduced by diffraction gratings to conduct measurements of the spectral line shape of SXRL amplifiers in a single shot. The concept of such powerful instrument was proposed by Chilla et al. [18], but it had never demonstrated to date.

The experimental setup of the interferometer is shown in Fig. 1(a). Its geometry is a variation of that corresponding to the Mach-Zehnder interferometers we have used extensively in the diagnostics of dense plasmas, in which diffraction gratings are used to split the beam [ $\underline{6}-11]$. The key difference between the two instruments is that in the interferometer used for plasma diagnostics the time delay introduced across the first diffraction-order beam is compensated when the two arms of the interferometer are recombined in the second grating. In contrast, in the configuration described in this Letter, the second grating doubles the time delay, which makes the measurement of a linewidth in a single shot possible. The instrument of interest here consists of two flat diffraction gratings (G1 and G2) positioned at grazing incidence to act as beam splitters and a set of four grazing incidence mirrors (M1-M4), two in each of the arms of the interferometer. The zero- and first-order beams from the first grating are redirected by reflections from mirrors (M1-M4) to emerge collinearly after diffraction on the second grating (G2). The interference fringe pattern produced is recorded with a back-thinned CCD array detector. The SXRL beam exiting the laser amplifier is collimated by a near normal-incidence Mo-Si multilayer spherical mirror S2 with a focal length of $f=37.5 \mathrm{~cm}$ 
a

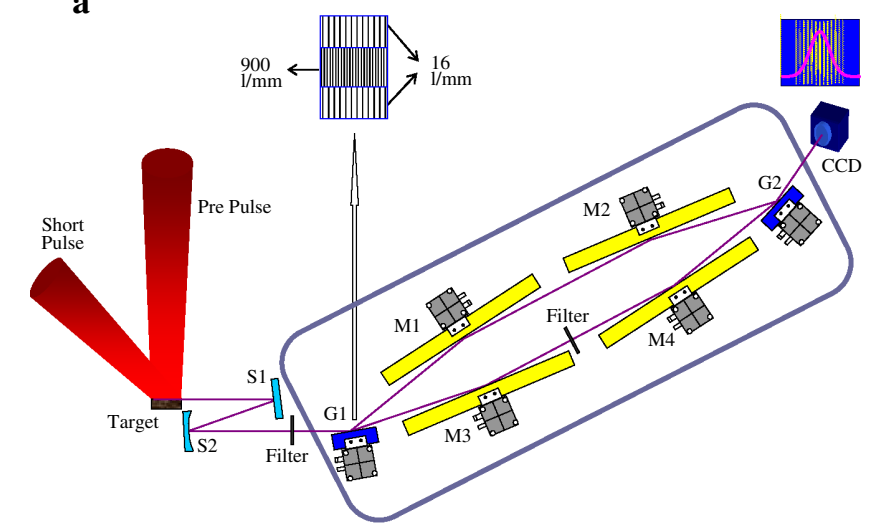

b

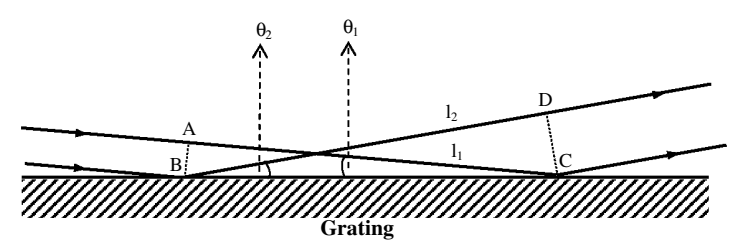

Fig. 1. (a) Single-shot SXRL linewidth measurement interferometer setup. The SXRL enters the interferometer from left side. The SXRL target is a $4 \mathrm{~mm}$ wide Pd slab; S1, S2: normal-incidence Mo-Si mirrors, $\mathrm{S} 1$ is flat mirror, S2 is spherical mirror, $f=37.5 \mathrm{~cm}$; G1, G2: flat gold coated diffraction gratings; M1-M4: gold coated grazing incidence mirrors; Filter: $0.3 \mu \mathrm{m}$ thick $\mathrm{Zr}$ filter. (b) Wavefront delay introduced by the diffraction grating. The input beam diameter is $L=\mathrm{AB} ; \theta_{1}$ is the input grazing incidence angle; $\theta_{2}$ is the output angle measured from grating surface. $l_{1}-l_{2}$ is path difference, where $l_{1}=\mathrm{AC}, l_{2}=\mathrm{BD}$.

and is directed toward the first grating of the interferometer with the aid of a second Mo-Si mirror. The collimated beam is about $3.4 \mathrm{~mm}$ diameter at the location of the first grating. A $0.3 \mu \mathrm{m}$ thick $\mathrm{Zr}$ filter is placed at the input of the interferometer to block visible/ultraviolet plasma light from reaching the CCD detector.

The fringe visibility, $V=\left(I_{\max }-I_{\min }\right) /\left(I_{\max }+I_{\min }\right)$, is obtained by integrating the fringe intensity along the direction of the interference lines after subtracting background. A time delay between the wavefront of the zero- and first-order beams is introduced when the pulse is diffracted by the two gratings (G1 and G2) [18], and reflected by the two grazing incidence mirrors, as illustrated in Fig. 1(b). Here $L$ is the input beam diameter ( $L=\mathrm{AB}), \theta_{1}$ is grazing incidence angle on grating $\mathrm{G} 1$ ( $3^{\circ}$ in our experiment), and $\theta_{2}$ is the output angle. Grating G1 introduces a tilt in the wavefront that produces a time delay across the beam relative to the zero-order beam. The second grating doubles the delay when recombining the two interferometer arms into a collinear path. The time delay is given by

$$
\Delta t=2\left(l_{1}-l_{2}\right) / c=2 L \delta \lambda /\left(c \sin \left(\theta_{1}\right)\right),
$$

where $l_{1}=\mathrm{AC}, l_{2}=\mathrm{BD}$ [Fig. 1(b)], $c$ is the speed of light, $\delta$ is the grating groove density (900 lines $/ \mathrm{mm}$ ), and $\lambda$ the laser wavelength. The interferogram is captured by the CCD camera with a $13.5 \mu \mathrm{m}$ square pixel array. For our soft x-ray laser wavelength $\lambda=14.7 \mathrm{~nm}$ one pixel corresponds to a time delay of 22.8 fs. The incidence angle onto the grating G1 was selected to split the energy evenly between the zero and the first diffraction orders. For this grating, this occurs at an angle of about $6^{\circ}$ [7]. However, an angle of $3^{\circ}$ was chosen instead to ensure that the time delay is sufficient to record the complete visibility curve. To compensate for the difference in beam intensity caused by this smaller angle, a $0.3 \mu \mathrm{m}$ thick $\mathrm{Zr}$ filter was inserted in the zero-order arm of the interferometer. This causes both beams to have similar intensity on the CCD, which is essential for generating fringes with high visibility. The straightness and uniformity of the fringes is strongly influenced by the flatness of the mirrors. The use of insufficiently flat optics was observed to strongly deteriorate the fringes. The grazing incidence mirrors used to obtain the results presented here were flat within $\lambda / 10$ (peak to valley $\lambda=632.8 \mathrm{~nm}$ ). The alignment of the interferometer was facilitated using a semiconductor laser. For this purpose, we used custom-made gratings designed to have three vertically staggered ruling zones [inset in Fig. 1(a)]. The central zone is ruled with a groove density of 900 lines/mm to diffract the soft x-ray beam in the center of the substrate. The top and the bottom regions are ruled with a groove density of 16 lines/mm designed to diffract along the same path of the SXRL an infrared laser diode with a similar coherence length. The chosen laser diode has a central wavelength of $827 \mathrm{~nm}$ and an estimated coherence length of $\sim 260 \mu \mathrm{m}$. The three-stripe ruling design allows for the rotation ( $180^{\circ}$ flip) of the second grating in order to reverse the blaze angle direction as needed to recombine the two interferometer's arms with similar efficiency to assure a good fringe visibility onto the CCD.

Figures 2(a) and 2(b) show two interferograms corresponding to the $\lambda=827 \mathrm{~nm}$ diode laser acquired for two different injected currents. The second row shows lineouts corresponding to the integration of the intensity of the fringes along the vertical direction, and the third row illustrates the fringe visibilities calculated from the integration data. The CCD used to capture the image of the fringes produced by the diode laser has a pixel size of $22.5 \mu \mathrm{m} / \mathrm{pixel}$, that for the $4 \times$ magnification used on the experiment corresponds to a time delay of $9.5 \mathrm{fs}$ per pixel. The linewidth of the diode laser changes with
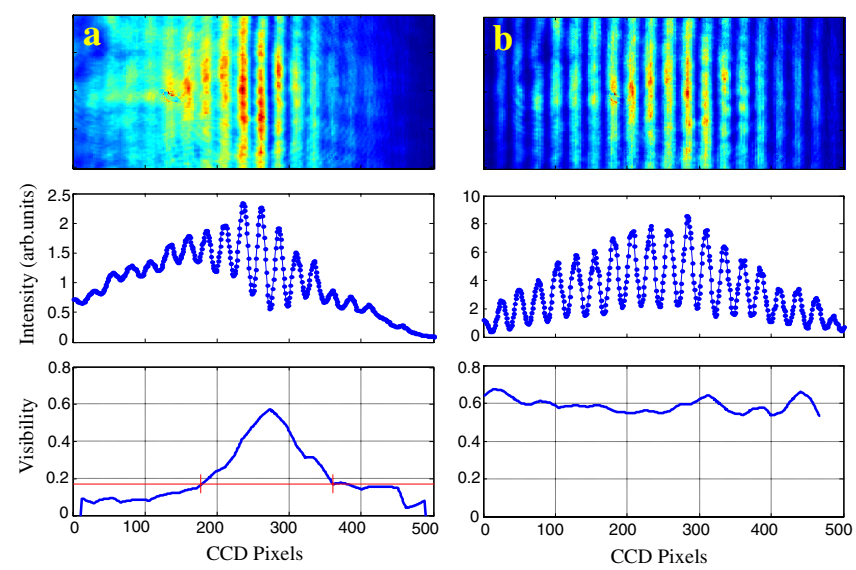

Fig. 2. Interferograms corresponding to a $\lambda=827 \mathrm{~nm}$ diode laser for two different injected currents: (a) $22 \mathrm{~mA}$ and (b) $30 \mathrm{~mA}$. The linewidth of the diode laser is observed to narrowed from $1.5 \mathrm{~nm}$ to less than $0.5 \mathrm{~nm}$. 
the injected current. Consequently, changing the current is a convenient way to check the ability of the instrument to measure different linewidths by comparison with measurements obtained with an optical spectrograph. Figure 2(a) shows the result obtained for a diode current of $22 \mathrm{~mA}$. The measured coherence length is $259 \mu \mathrm{m}(1 / e$ half-width), which corresponds to a linewidth of $1.4 \mathrm{~nm}$ FWHM. Figure 2(b) shows the result corresponding to a diode current of $30 \mathrm{~mA}$. In this case the visibility is almost flat, corresponding to a very narrow linewidth of less than $0.5 \mathrm{~nm}$. These measurements were found to be in good agreement with those performed using a grating spectrograph.

The $\lambda=14.7 \mathrm{~nm}$ Pd soft x-ray laser beam was generated by amplification of spontaneous emission in a transient population inversion created between the $4 d^{1} S_{0}-4 p^{1} P_{1}$ levels of Ni-like $\mathrm{Pd}$ by collisional electron impact excitation in a laser-created plasma $[\underline{19}, \underline{20}]$. A line focus plasma was produced by heating a $4 \mathrm{~mm}$ long $\mathrm{Pd}$ slab target at grazing incidence [21-23] with a highintensity optical laser pulse from a chirped pulse amplified Ti:Sapphire laser system. A sequence of two 200 ps FWHM duration pre-pulses with energies of 40 and $400 \mathrm{~mJ}$ separated by a time delay of $7 \mathrm{~ns}$ were focused at normal incidence onto the target to form a $30 \mu \mathrm{m}$ wide by $4 \mathrm{~mm}$ long line pre-plasma with a large density of $\mathrm{Ni}$ like ions. After a $400 \mathrm{ps}$ delay from the peak of the second pre-pulse, a 5 ps FWHM duration heating pulse of $700 \mathrm{~mJ}$ energy was made to impinge onto the plasma at a grazing incidence angle of $23^{\circ}$ in an overlapping line focus, resulting in transient population inversion and strong laser amplification in the $\lambda=14.7 \mathrm{~nm}$ line of Ni-like Pd.

A single-shot soft x-ray laser interferogram for the $\mathrm{Ni}$ like Pd laser is shown in Fig. 3. A Gaussian function fits very well the measured visibility curve. This can be expected, because while the atomic line profile is defined by a Voigt profile, gain narrowing during amplification causes the lineshape to approach a Gaussian [24]. A
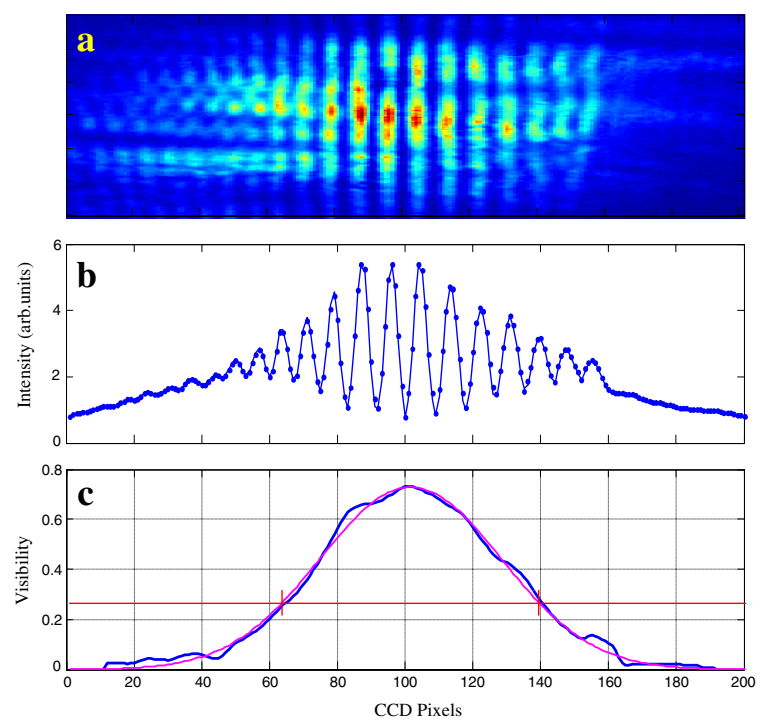

Fig. 3. Single shot interferogram for a transient collisional Nilike $\mathrm{Pd}$ laser at $\lambda=14.7 \mathrm{~nm}$. The coherence length is measured to be $260 \mu \mathrm{m}$ : (a) interferogram, (b) integration of the fringe intensity along the vertical direction, and (c) measured fringes visibility curve (blue) and Gaussian fit (magenta).
Gaussian fit to the fringe visibility yields a coherence length of $260 \mu \mathrm{m}$ (1/e half-width). To verify that the spectral shape is the same over the entire far field beam profile, which in this type of lasers is defined by multiple speckle spots, we compared the results from the vertical integration of the fringes in several different regions with good visibility, each defined by a horizontal stripe containing a fraction of the total beam area. The coherence length was found to be quite independent from the irregular intensity distribution of the beam (variation $<5 \%$ ). To further corroborate the result, we recorded several sets of measurements, each composed of up to 30 single-shots interferograms obtained at the same plasma irradiation condition. All the measurements gave similar values irrespective of shot-to-shot variation in the SXRL spatial profile, showing good reproducibility, and resulting in a coherence length of $(260 \pm 18) \mu \mathrm{m}(1 / e$ half-width). The corresponding linewidth of $\Delta \lambda / \lambda \approx 3.0 \times 10^{-5}$ is similar to that measured using multiple shots with a wavefront division interferometer for a transient collisional Ni-like Mo laser at $\lambda=18.9 \mathrm{~nm}$ excited at the same grazing incidence angle $\left(\Delta \lambda / \lambda \approx 3.3 \times 10^{-5}\right)$ [ 15$]$. To further validate the measurement, we conducted additional experiments using different fringe spacing. The fringe spacing can be easily adjusted by changing the tilt of the second collimation mirror S2 and the fringe orientation can be adjusted by rotating the second grating, G2. With a higher fringe density it is possible to obtain improved image sampling and higher spatial resolution, but the visibility is degraded if the fringe size becomes excessively small. In any case, the measured coherence length should be independent of the interference fringe period selected, which was verified by the experiments. Figure 4 displays three interferograms acquired for the same SX $\bar{R} L$ laser plasma conditions varying the fringe period. The coherence length was measured to remain almost unchanged at $\sim 260 \mu \mathrm{m}$ as the fringe period was changed from 15 pixels in Fig. 4(a) to 5 pixels in Fig. 4(c).

In conclusion, we have obtained single shot SXRL linewidth measurements using a grating interferometer that makes use of the time delay introduced by diffraction gratings. The coherence length of a Ni-like Pd transient
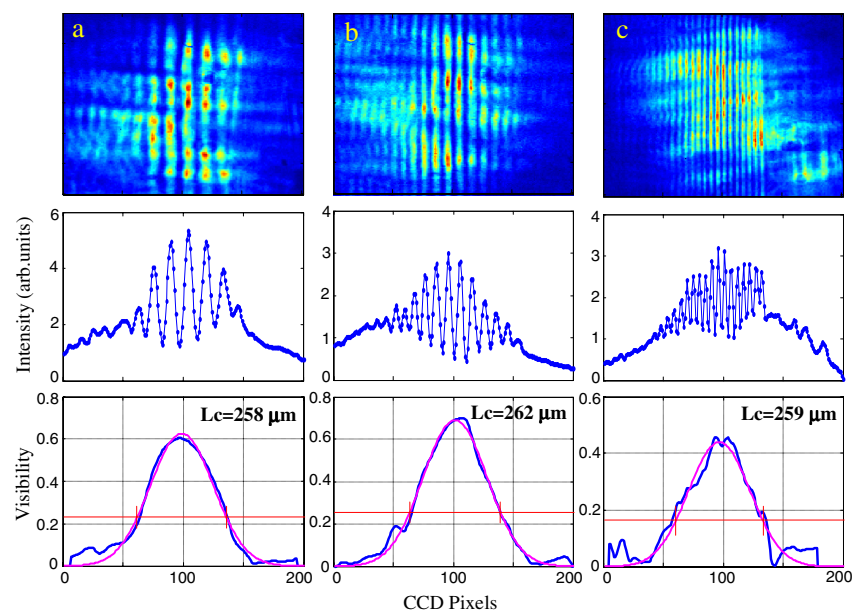

Fig. 4. Interferograms for Ni-like $\mathrm{Pd}$ laser $\lambda=14.7 \mathrm{~nm} \mathrm{ac}-$ quired corresponding to different fringes periods: (a) 15 pixels, (b) 10 pixels, and (c) 5 pixels. The measured temporal coherence length $\mathrm{Lc} \sim 260 \mu \mathrm{m}$, independent of the fringe spacing. 
collisional SXRL operating at $\lambda=14.7 \mathrm{~nm}$ was measured to be $(260 \pm 18) \mu \mathrm{m}$ (1/ $e$ half-width), corresponding to a linewidth of $\Delta \lambda / \lambda \sim 3.0 \times 10^{-5}$. This bandwidth could support a FWHM pulsewidth of 720 fs assuming a Gaussian line profile. This single-shot linewidth measurement can contribute to the development of the next generation of soft x-ray lasers.

We thank Oscar Martinez for useful discussions. This work was supported by the AMOS program of the Office of Basic Energy Sciences, U.S. Department of Energy, and by NSF Engineering Research Center Program using equipment developed under NSF Award MRI-ARRA 09-561, and in part under the auspices of the U.S. Department of Energy by Lawrence Livermore National Laboratory under Contract DE-AC52-07NA27344.

\section{References}

1. J. E. Trebes, S. B. Brown, E. M. Campbell, D. L. Matthews, D. G. Nilson, G. F. Stone, and D. A. Whelan, Science 238, 517 (1987).

2. P. W. Wachulak, M. C. Marconi, R. A. Bartels, C. S. Menoni, and J. J. Rocca, J. Opt. Soc. Am. B 25, 1811 (2008).

3. A. Isoyan, F. Jiang, Y. C. Cheng, F. Cerrina, P. Wachulak, L. Urbanski, J. J. Rocca, C. Menoni, and M. C. Marconi, J. Vac. Sci. Technol. B 27, 2931 (2009).

4. L. Urbanski, A. Isoyan, A. Stein, J. J. Rocca, C. S. Menoni, and M. C. Marconi, Opt. Lett. 37, 3633 (2012).

5. L. B. Da Silva, T. W. Barbee, Jr., R. Cauble, P. Celliers, D. Ciarlo, S. Libby, R. A. London, D. Matthews, S. Mrowka, J. C. Moreno, D. Ress, J. E. Trebes, A. S. Wan, and F. Weber, Phys. Rev. Lett. 74, 3991 (1995).

6. J. Filevich, K. Kanizay, M. C. Marconi, J. L. A. Chilla, and J. J. Rocca, Opt. Lett. 25, 356 (2000).

7. J. Filevich, J. J. Rocca, M. C. Marconi, R. F. Smith, J. Dunn, R. Keenan, J. R. Hunter, S. J. Moon, J. Nilsen, A. Ng, and V. N. Shlyaptsev, Appl. Opt. 43, 3938 (2004).

8. J. Filevich, J. J. Rocca, M. C. Marconi, S. J. Moon, J. Nilsen, J. H. Scofield, J. Dunn, R. F. Smith, R. Keenan, J. R. Hunter, and V. N. Shlyaptsev, Phys. Rev. Lett. 94, 035005 (2005).

9. M. Purvis, J. Grava, J. Filevich, M. C. Marconi, J. Dunn, S. J. Moon, V. N. Shlyaptsev, E. Jankowska, and J. J. Rocca, Phys. Rev. E 76, 046402 (2007).
10. J. Grava, M. A. Purvis, J. Filevich, M. C. Marconi, J. J. Rocca, J. Dunn, S. J. Moon, and V. N. Shlyaptsev, Phys. Rev. E 78, 016403 (2008).

11. M. A. Purvis, J. Grava, J. Filevich, D. P. Ryan, S. J. Moon, J. Dunn, V. N. Shlyaptsev, and J. J. Rocca, Phys. Rev. E 81, 036408 (2010).

12. Y. Wang, M. Berrill, F. Pedaci, M. Shakya, S. Gilbertson, Z. Chang, E. Granados, B. Luther, M. Larotonda, and J. J. Rocca, Phys. Rev. A 79, 023810 (2009).

13. D. S. Whittaker, M. Fajardo, Ph. Zeitoun, J. Gautier, E. Oliva, S. Sebban, and P. Velarde, Phys. Rev. A 81, 043836 (2010).

14. R. F. Smith, J. Dunn, J. R. Hunter, J. Nilsen, S. Hubert, S. Jacquemot, C. Remond, R. Marmoret, M. Fajardo, P. Zeitoun, L. Vanbostal, C. L. S. Lewis, M. F. Ravet, and F. Delmotte, Opt. Lett. 28, 2261 (2003).

15. L. M. Meng, D. Alessi, O. Guilbaud, Y. Wang, M. Berrill, B. M. Luther, S. R. Domingue, D. H. Martz, D. Joyeux, S. De Rossi, J. J. Rocca, and A. Klisnick, Opt. Express 19, 12087 (2011).

16. J. A. Koch, B. J. MacGowan, L. B. Da Silva, D. L. Matthews, J. H. Underwood, P. J. Batson, R. W. Lee, R. A. London, and S. Mrowka, Phys. Rev. A 50, 1877 (1994).

17. L. Urbanski, M. C. Marconi, L. M. Meng, M. Berrill, O. Guilbaud, A. Klisnick, and J. J. Rocca, Phys. Rev. A 85, 033837 (2012).

18. J. L. A. Chilla, J. J. Rocca, O. E. Martinez, and M. C. Marconi, Opt. Lett. 21, 955 (1996).

19. J. Dunn, Y. Li, A. L. Osterheld, J. Nilsen, J. R. Hunter, and V. N. Shlyaptsev, Phys. Rev. Lett. 84, 4834 (2000).

20. B. M. Luther, Y. Wang, M. A. Larotonda, D. Alessi, M. Berrill, J. J. Rocca, J. Dunn, R. Keenan, and V. N. Shlyaptsev, IEEE J. Quantum Electron. 42, 4 (2006).

21. Y. Wang, M. A. Larotonda, B. M. Luther, D. Alessi, M. Berrill, V. N. Shlyaptsev, and J. J. Rocca, Phys. Rev. A 72, 053807 (2005).

22. R. Keenan, J. Dunn, P. K. Patel, D. F. Price, R. F. Smith, and V. N. Shlyaptsev, Phys. Rev. Lett. 94, 103901 (2005).

23. B. M. Luther, Y. Wang, M. A. Larotonda, D. Alessi, M. Berrill, M. C. Marconi, J. J. Rocca, and V. N. Shlyaptsev, Opt. Lett. 30, 165 (2005).

24. D. Benredjem, C. Möller, J. Dubau, and T. Ball, Phys. Rev. A 73, 063820 (2006). 